\title{
Modelo de ecuaciones estructurales, alternativa para medir el fenómeno de las mujeres STEM en México
}

\author{
Structural Equation Models: An Alternative to Measure the Phenomenon of \\ STEM Women in Mexico
}

\begin{abstract}
Modelo de equações estruturais, alternativa para medir o fenômeno das mulheres STEM no México
\end{abstract}

\author{
Claudia Alejandra Hernández Herrera \\ Instituto Politécnico Nacional, México \\ cahernandezh@ipn.mx \\ http://orcid.org/0000-0002-4060-2941
}

\section{Resumen}

Aunque cada vez hay más mujeres en las universidades en las áreas de ciencia, tecnología, ingeniería y matemáticas (STEM), en términos de representatividad aún están lejos de igualar a los hombres. Los estereotipos de género y la segregación de las carreras STEM son obstáculos para las estudiantes, ya que generan prácticas excluyentes y ambientes hostiles. El objetivo de este artículo fue proponer un modelo de medición del fenómeno de las mujeres STEM que considerara variables latentes relacionadas con las creencias masculinas, las inspiraciones, las estrategias de afrontamiento, el mercado laboral, la escuela y el gusto por las matemáticas. Para ello, se construyó un instrumento que mide la percepción de las mujeres STEM en universitarios. Se aplicaron 1826 cuestionarios a estudiantes de dos escuelas de educación superior del Instituto Politécnico Nacional. Se realizó el análisis factorial exploratorio y confirmatorio, posteriormente se trabajó el modelo de ecuaciones estructurales y por último se establecieron seis hipótesis generadas de las variables latentes. Se encontró que el afrontamiento contribuye a explicar la variable Escuela, además de que las creencias masculinas explican los constructos Escuela y Mercado Laboral. Se concluye que es necesario que las escuelas trabajen para desarrollar ambientes respetuosos y 


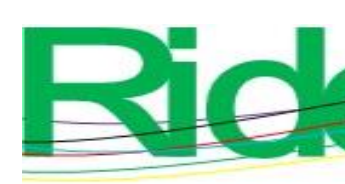

\section{Revista Iberoamericana para la Investigación y el Desarrollo Educativo ISSN $2007-7467$}

um instrumento que mede a percepção de mulheres STEM em estudantes universitários. 1826 questionários foram aplicados a alunos de duas escolas de ensino superior do Instituto Politécnico Nacional. Foi realizada a análise fatorial exploratória e confirmatória, posteriormente trabalhado o modelo de equações estruturais e, por fim, estabelecidas seis hipóteses geradas a partir das variáveis latentes. Verificou-se que o coping contribui para explicar a variável Escola, além do fato de que as crenças masculinas explicam os construtos Escola e Mercado de Trabalho. Conclui-se que é necessário que as escolas trabalhem para desenvolver ambientes respeitosos e desafiadores para as mulheres, somado ao fato de que o setor de trabalho tem que se apoiar para fechar as lacunas e eliminar a discriminação de gênero.

Palavras-chave: crenças masculinas, ensino superior, modelo de equações estruturais, mulheres STEM.

Fecha Recepción: Diciembre 2020

Fecha Aceptación: Junio 2021

\section{Introducción}

Es evidente que la presencia de las mujeres está desdibujada en los campos de la ciencia, tecnología, ingeniería y matemáticas (STEM, por sus siglas en inglés). Se trata de una problemática multifactorial que ha llevado a las mujeres a descartar desde temprana edad, desde la infancia, la posibilidad de desenvolverse en estas áreas del conocimiento (Organización de las Naciones Unidas para la Educación, la Ciencia y la Cultura [Unesco], 2019). La discriminación de género, los sesgos, los estereotipos, las normas sociales, la escasa motivación y el poco reconocimiento a sus habilidades son algunas de las razones por las que las mujeres no incursionan, permanecen o son promovidas en los ámbitos de STEM. Si bien implican caminos que pueden ser difíciles de recorrer y no aptos para cualquiera, sin importar el género, muchas jóvenes descartan prematuramente las STEM debido a la falsa creencia de que calzan mejor a los hombres y a que, por ser tan demandantes profesionalmente, impiden encontrar un equilibrio entre el trabajo y la familia, ambos supuestos altamente imantados por estereotipos de género (van Tuijl y van der Molen, 2016). En efecto, a los hombres adolescentes se les recomienda más estudiar ingeniería que a las mujeres puesto que se les atribuye mayor habilidad matemática (Pozo et al., 2020).

A pesar de la falta de ingenieros en diferentes áreas, los niños entre 10 y 14 años, aunque especialmente las niñas, no muestran interés por adentrarse en esas carreras, ya que la gran mayoría piensa que se requiere de una inteligencia superior para ello (Poniszewska, 


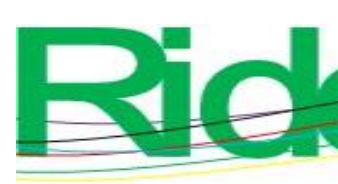

Revista Iberoamericana para la
Investigación y el Desarrollo Educativo
ISSN $2007-7467$

Szukalska y Wilczyński, 2021). Es desalentador que la existencia de prejuicios de género provoque brechas en la participación de las mujeres en los campos de STEM. Y por las mismas razones, las mujeres proyectan menos sentido de pertinencia, lo que perjudica sus aspiraciones en esos campos (Moss, Sanzari, Caluori y Rabasco, 2018).

La literatura enfatiza en que las diferencias de género en las carreras de STEM están relacionadas con la baja autoestima de las mujeres, además de una pobre percepción de sus habilidades en los campos de las matemáticas, escasas expectativas de pertinencia social y creencias de una baja autoeficacia. Otro aspecto a considerar es que los maestros suelen considerar que las matemáticas requieren brillantez y, lamentablemente, tienden a pensar que las niñas carecen de esa genialidad (Copur, Thacker y Quinn, 2020; Rebollo y De la Rica, 2020; Tellhed, Bäckström y Björklund, 2017; van Langen, 2015; Wang y Degol, 2017).

De acuerdo con Gunderson, Ramirez, Levine y Beilock (2012), prolifera la preocupación de que las niñas en diversas ocasiones tienen actitudes negativas hacia las matemáticas, incluso llegan a desarrollar mayores autoconceptos nocivos y ansiedades hacia dichas áreas que los niños; lo anterior, una vez más, está relacionado con los estereotipos de género. El impacto sobre el concepto que tienen las niñas de ellas se refleja en su desempeño hacia las matemáticas y la búsqueda de trayectorias profesionales relacionadas con los campos de STEM. Las expectativas que tienen los padres y los profesores sobre las competencias matemáticas están sesgadas e influyen en el rendimiento académico y las actitudes de los niños.

Algo igualmente lamentable es que existen estudios que señalan que a las mujeres se les percibe como personas que carecen de cualidades necesarias para ser científicas exitosas, lo que contribuye a la discriminación. Además, se piensa que son menos inteligentes y competentes (Carli, Alawa, Lee, Zhao y Kim, 2016; Eaton, Saunders, Jacobson y West, 2020). La literatura enfatiza en que las mujeres jóvenes tienen menores posibilidades de persistir en la ciencia que los hombres; también señala que estos tienen más recursos familiares que aquellas, por lo que suelen tener más experiencias científicas (Hanson, 1996).

Según el Global Gender Gap Report 2020 realizado por el Foro Económico Mundial [WEF, por sus siglas en inglés] (2020), América Latina cuenta con un índice de brecha de género global del 0.721. La participación económica de las mujeres es de 0.642 y el de empoderamiento político de 0.269 . Es de destacar que solo $43 \%$ de las mujeres mexicanas participa en el mercado laboral, de las cuales $26 \%$ cuenta con un empleo de medio tiempo. A pesar de contar con la misma formación académica, las situaciones profesionales de los 


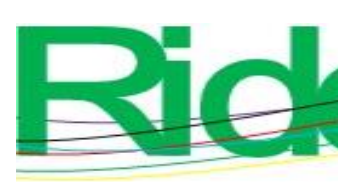

Revista Iberoamericana para la
Investigación y el Desarrollo Educativo
ISSN $2007-7467$

graduados difieren según el género. Las mujeres experimentan mayores obstáculos para encontrar un trabajo, tienen menos posibilidades de hallar un empleo acorde a su educación y lo más grave es que en diversos puestos ellas ganan menos (Jasko, Pyrkosz, Czarnek, Dukała y Szastok, 2020). López, Grazzi, Guillard y Salazar (2018) subrayan que existen diversidad de obstáculos para las mujeres que frenan su integración en los campos de STEM. Si bien se ha tenido un avance que ha permitido reducir las brechas de género, todavía existen dificultades para alcanzar puestos de liderazgo en diversas esferas de la ciencia, tecnología y la innovación. Respecto a las ocupaciones laborales en ciencia y tecnología, México tiene diferencias importantes en aquellos puestos de niveles altos y medios. En comparación con el área de ciencias de la salud, donde $70 \%$ de las mujeres está ocupada y entre $40 \%$ y $45 \%$ está en puestos altos y medios, en el área de tecnologías de la información y la comunicación (TIC), en ciencias e ingeniería solo $17 \%$ de mujeres ocupa puestos altos y $6 \%$ puestos medios. Cabe señalar que lo puestos medios no requieren educación terciaria, pero los puestos altos sí necesitan ese tipo de educación (López et al., 2018).

Aunque las mujeres que estudian en campos de STEM no experimentan de forma abierta la discriminación, en ocasiones perciben microagresiones que las llevan a sentirse en entornos amenazantes. Estos eventos llevan a que las mujeres se desconecten psicológicamente, lo que impacta en su desempeño, su motivación y su autoestima. Como sabemos, la autoestima permite desarrollar altos niveles de satisfacción y resistencia ante el agotamiento (Casad, Petzel e Ingalls, 2019).

El objetivo de este artículo es proponer y confirmar un modelo de medición del fenómeno de las mujeres en STEM que considere las siguientes variables latentes detectadas en la literatura: las creencias masculinas, las inspiraciones, estrategias de afrontamiento, el mercado laboral, la escuela y el gusto por las matemáticas. Lo anterior para determinar las relaciones causales sobre datos no experimentales por medio de un modelo de ecuaciones estructurales.

\section{Marco teórico del fenómeno STEM}

Las mujeres STEM es un fenómeno multivariable. Hasta ahora se ha hecho énfasis en las creencias que se generan a partir de las ideologías provocadas por los estereotipos tradicionales de género. De igual forma, se ha trabajado sobre el discurso que se propaga en el entorno escolar, además de las técnicas de enfrentamiento desarrolladas por las mujeres, aunado al reconocimiento temprano de sus habilidades en matemáticas, sus inspiraciones y 


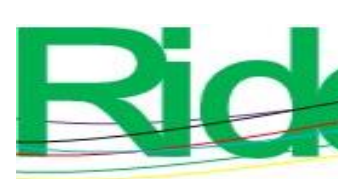

Revista Iberoamericana para la Investigación y el Desarrollo Educativo ISSN $2007-7467$

los diversos obstáculos a los que se enfrentan en el mercado laboral. Ahora bien, Carli et al. (2016) mencionan que a los hombres se les considera como líderes, analíticos, competitivos e independientes, mientras que a las mujeres amables, comprensivas, cálidas y serviciales. Lo anterior ha suscitado una diversidad de debates académicos que reafirma que la escasa paridad de hombres y mujeres en los campos de STEM se ha generado por las ideologías de género que apoyan la idea de que unos y otras tienen habilidades y aptitudes diferentes. En cuanto a las mujeres, se cree que carecen de las fortalezas necesarias para los campos de STEM, aunado a que las niñas muestran poco interés por este y deciden optar por carreras que mejor encajan socialmente (Pološki, Obadić y Sinčić, 2019). Sin duda, los sesgos de género benefician a los varones, ya que se consideran mucho más competentes que las mujeres (Eaton et al., 2020).

El sexismo benevolente se manifiesta mediante conductas condescendientes hacia las mujeres que generan en ellas bajas expectativas y pobres estándares de excelencia, y provoca que, para conseguir ser aceptadas en esos campos masculinizados, actúen más como hombres y realicen esfuerzos extras para poder sentir que son competitivas (Makarova, Aeschlimann y Herzog, 2016).

El estudio de la autoeficacia surge para indagar sobre el comportamiento de los jóvenes en los campos de STEM; aspira a predecir el tiempo que un estudiante persistirá en alguna actividad para resolverla. La autoeficacia es definida como la confianza sobre las habilidades y capacidades para tener éxito en determinadas tareas (Paunonen y Hong, 2010). Elementos como el autoconcepto, el valor que se le da a las tareas, la autonomía, la afinidad y la mentalidad de crecimiento son sumamente relevantes para el desarrollo del compromiso, el desempeño, la selección de materias y las aspiraciones que llegan a tener los estudiantes de STEM (Murphy, MacDonald, Wang y Danaia, 2019). De hecho, Tellhed et al. (2017) sostienen que la autoeficacia permite identificar los intereses por las carreras de STEM.

Y en esa línea, Zander, Höhne, Harms, Pfost y Hornsey (2020) comentan que las niñas, a pesar de tener calificaciones en matemáticas similares a los niños, presentan una menor autoeficacia y muestran tener menor autoestima; en contraste, los niños poseen sentimientos positivos. Existe evidencia de que las niñas tienen un autoconcepto más bajo en STEM y, por ello, es posible que mantengan un bajo interés y atribuyan cualquier fracaso en estos campos a la falta de capacidad (Murphy et al., 2019). De forma análoga, el trabajo de Heyder, Weidinger y Steinmayr (2020) apunta que las mujeres muestran una menor capacidad del desarrollo de su autoconcepto y, por ende, baja motivación intrínseca en 


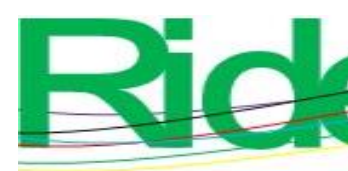

Revista Iberoamericana para la Investigación y el Desarrollo Educativo ISSN $2007-7467$

matemáticas, mientras que con los varones pasa lo contrario. La motivación puede ser afectada por la construcción de roles y estereotipos de género que se reproducen en la sociedad y debe de considerarse como importante, ya que permite incrementar el rendimiento académico (Jungert, Hubbard, Dedic y Rosenfield, 2019; Yang y Gao, 2019).

Respecto a por qué las mujeres presentan menor confianza en las matemáticas, Sax, Kanny, Riggers, Whang y Paulson (2015) sostienen que el autoconcepto matemático representa un predictor de la motivación, las emociones y el desempeño de los individuos y ayuda a explicar el papel del yo en la escuela. Lo anterior se entrecruza con las autopercepciones (que se relacionan a su vez con el comportamiento del logro), a saber, las percepciones que tiene un estudiante de sus habilidades para las matemáticas con respecto a la de los demás. Además, el autoconcepto matemático tiene una fuerte relación con el rendimiento académico, por lo que es el encargado de darle forma a las aspiraciones en los campos de STEM. Indudablemente, el autoconcepto matemático influye demasiado en las mujeres. Para que aspiren con mayor frecuencia a estar en los campos de STEM, hay que trabajar en su confianza $\mathrm{y}$, sobre todo, evitar que las niñas subestimen su capacidad matemática. Según Koul, Lerdpornkulrat y Poondej (2017), a diferencia de las mujeres, en el caso de los varones las aspiraciones son independientes del autoconcepto matemático.

Por otro lado, de acuerdo con Christman y McClellan (2012), la resiliencia es un mecanismo interno que genera que las personas desarrollen rasgos positivos como la paciencia, la autodeterminación, la responsabilidad y el asumir riesgos con el objetivo de superar la adversidad. También activa la resistencia, la autoestima, la autoeficacia, la capacidad intelectual y la autonomía, y requiere de la combinación de otros elementos como el apoyo de los padres y mentores. Al respecto, Skolnik (2015) señala que otras estrategias para reforzar la resiliencia es trabajar en la fe, la automotivación, el apoyo familiar, tomar clases extras y trabajar de la mano con los profesores. Mientras que Thiry (2019) menciona que las habilidades individuales de afrontamiento, además del interés por el campo, la asertividad y la voluntad de saber aceptar la crítica y perder el miedo a equivocarse, son elementos que apoyan a las mujeres a persistir. Precisamente, Deb (2018) hace hincapié en que las mujeres deben desarrollar mecanismos de protección para hacerle frente a la adversidad y desarrollar conductas resilientes. Y considera la autoeficacia como un amortiguador interno y las relaciones sólidas y el apoyo de la comunidad como externos. Por último, Grau y Martinez (2017) expresaron que el elemento más importante para muchas mujeres para persistir en las carreras de STEM fue el apoyo familiar que recibieron. 

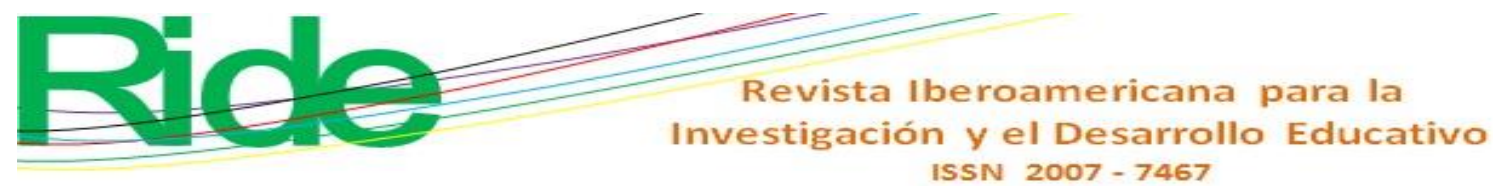

Los trabajos realizados por la feminista De Beauvoir (1981) son imprescindibles para entender el fenómeno de la subrepresentación de las mujeres en STEM. Esta pensadora explica que los hombres y las mujeres nunca han compartido la idea de imaginar un mundo basado en la igualdad. A pesar de los esfuerzos, ellas continúan sufriendo muchas desventajas. En materia económica, las mujeres y los hombres son sujetos completamente distintos: los hombres tienen salarios más altos, más oportunidades de triunfar, están presentes con mayor fuerza en la industria, la política, aunado a que poseen los mejores puestos. Las brechas de género en el mercado laboral son el producto de la formación familiar, elementos psicológicos, normas culturales y sociales (Bertocchi y Bozzano, 2020).

La literatura científica desarrollada hasta este momento da cuenta que para poder comprender la discriminación de género en el mercado laboral es necesario entender factores como la desigualdad en los ingresos, los niveles de educación, la distribución ocupacional y las oportunidades que tienen las mujeres en los sectores y la pobreza. Lo anterior aunado a que ciertas creencias de los roles de género influyen en las preferencias de los empleadores e incrementan la brecha de género (Miluka, 2013). Los beneficios económicos están relacionados con el rendimiento académico y el género. Los hombres obtienen mejores ganancias que las mujeres en los campos de STEM, a pesar de que ellas, en varios casos, cuentan con mejor preparación académica (Olitsky, 2014). Por otra parte, los programas de STEM orientados exclusivamente para mujeres han tenido un efecto positivo en las aspiraciones de estas (Szelényi e Inkelas, 2011).

Según van Veelen, Derks y Endedijk (2019), en diversas ocasiones las mujeres STEM se enfrentan a un doble problema en el mercado de trabajo: el primero es que tienen que trabajar en ambientes en donde son superadas en número por los varones y el segundo es que las mujeres en esos campos son estereotipadas de forma negativa y esa amenaza a la identidad de género influye de forma negativa en el compromiso laboral y la confianza profesional de las mujeres.

En la actualidad, persiste una disparidad salarial alarmante en los ámbitos STEM entre las mujeres y los hombres que ostentan los mismos niveles académicos. La inequidad de género en los campos laborales puede llegar a disuadir fuertemente a las mujeres que han obtenido títulos y grados académicos para ingresar, avanzar y perseverar en los escenarios dominados por hombres (Okahana y Hao, 2019). Investigaciones realizadas por Castaño, Lubiano y García (2020) y Schlenker (2015) indican que las mujeres que son madres trabajan más horas en los campos de STEM; a pesar de ello, tienen mayor probabilidades de estar 


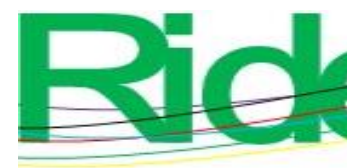

\section{Revista Iberoamericana para la Investigación y el Desarrollo Educativo ISSN $2007-7467$}

fuera de la fuerza laboral. La segregación laboral es un problema de igualdad de género que acarrea serias consecuencias que pueden orillar a las mujeres a vivir en pobreza. De acuerdo con Yamaguchi (2019), las formas en las que se manejan los mercados laborales están relacionadas con la persistente idea de la distribución de actividades domésticas que existen en el hogar y que siguen estando a cargo de las mujeres.

Lamentablemente, es menos probable que una mujer sea contratada para trabajos considerados como masculinos y más posible que sea elegida en aquellos empleos relacionados con lo femenino. Esto refuerza la penalización de género y acentúa la tipificación sexual de las ocupaciones.

En los campos de STEM las divisiones del trabajo por género continúan existiendo. El liderazgo sigue siendo visto como una cualidad exclusiva del género masculino. La errónea idea de que los hombres son los actores principales en los procesos y que las mujeres solo son el apoyo emocional no ha parado de replicarse. En definitiva, las mujeres están en desventaja al tener posiciones de menor poder. En este tipo de organizaciones masculinizadas incluso en ocasiones son vistas como extrañas (Acker, 2005). Es sabido que la brecha salarial generacional en el caso de las mujeres desaparece cuando se controlan variables individuales como la maternidad y el color de piel; en contraste, para los hombres las diferencias salariales se controlan respondiendo a las características del mercado (Manzoni, 2019; Varela, 2019).

Con base en la revisión de la literatura, se proponen las siguientes hipótesis:

- $\quad$ H1: el gusto por las matemáticas aporta a la variable Escuela.

- $\quad \mathrm{H} 2$ : las técnicas de afrontamiento aporta al constructo Escuela.

- $\quad$ H3: las creencias masculinas aportan a explicar la variable latente Escuela.

- H4: las creencias masculinas aportan a explicar el mercado laboral.

- $\quad$ H5: las inspiraciones aportan a explicar el mercado laboral.

- H6: la escuela aporta a comprender el mercado laboral.

\section{Materiales y métodos}

Es una investigación cuantitativa y transversal. El estudio se realizó en el mes de septiembre de 2019. Se construyó un instrumento que pretende medir la percepción del fenómeno STEM en las instituciones de educación superior. Se trabajó con el marco teórico relacionado con las mujeres STEM. Se empleó la escala tipo Likert, en donde 1 es igual a totalmente en desacuerdo y 5 a totalmente de acuerdo; se trabajaron 22 ítems. Se aplicaron 

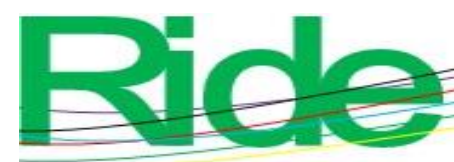

Revista Iberoamericana para la Investigación y el Desarrollo Educativo

ISSN $2007-7467$

los cuestionarios en dos escuelas que pertenecen al Instituto Politécnico Nacional y que cuentan con carreras del área de STEM. Se logró obtener 1826 cuestionarios; la participación de 776 mujeres y 1050 hombres. Para su aplicación se entrenaron a jóvenes del programa de créditos para actividades extracurriculares, quienes fueron los responsables del repliegue en las dos unidades académicas. La muestra fue no probabilística y por conveniencia. Se trabajó el análisis factorial exploratorio y el confirmatorio, ambos fueron de máxima verosimilitud. En el caso del análisis factorial exploratorio, se trabajó la rotación ortogonal promax, ya que esta se aplica más a los modelos reflectivos que consideran las percepciones de variables que trabaja con escalas. En la prueba estadística de Kaiser Meyer Olkin (KMO) se encontró 0.823 $>0.05$, lo que indica que los enunciados tienen capacidad de agrupación y poseen consistencia interna.

El análisis de las correlaciones permitió quitar del modelo los enunciados que no aportaban a las variables latentes. La matriz patrón se expone en la tabla 1. En total, se obtuvieron seis dimensiones. 
Tabla 1. Matriz patrón

\begin{tabular}{|c|c|c|c|c|c|c|}
\hline Variable/indicador & 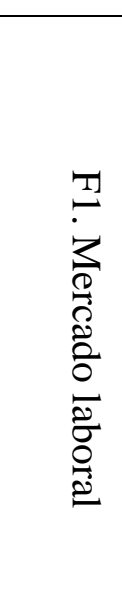 & 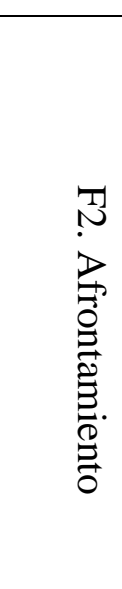 & 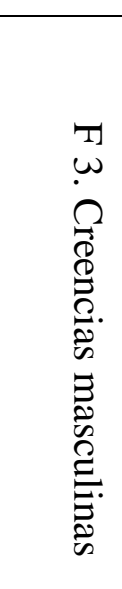 & 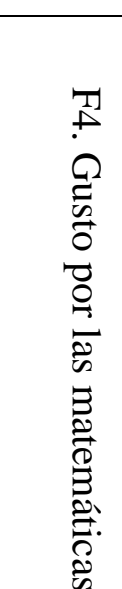 & 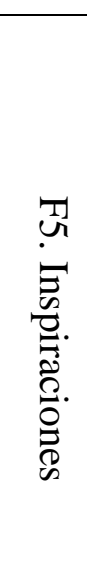 & $\begin{array}{l}T \\
2 \\
17 \\
2 \\
0 \\
\frac{0}{2} \\
2\end{array}$ \\
\hline $\begin{array}{l}\text { G1. Gusto más por las matemáticas } \\
\text { que por otras asignaturas. }\end{array}$ & & & & 0.878 & & \\
\hline $\begin{array}{l}\text { G2. Creencia sobre las posibles } \\
\text { aplicaciones de las matemáticas en la } \\
\text { vida real y en los campos de STEM. }\end{array}$ & & 0.324 & & & & \\
\hline $\begin{array}{l}\text { A3. En la escuela se ha trabajado con } \\
\text { problemas prácticos en donde se } \\
\text { proponen soluciones haciendo uso de } \\
\text { las áreas de STEM. }\end{array}$ & & 0.346 & & & & \\
\hline $\begin{array}{l}\text { C4. Pienso que los hombres son } \\
\text { mejores en las matemáticas que las } \\
\text { mujeres. }\end{array}$ & & & 0.854 & & & \\
\hline $\begin{array}{l}\text { C5. Pienso que los hombres son } \\
\text { mejores científicos que las mujeres. }\end{array}$ & & & 0.956 & & & \\
\hline $\begin{array}{l}\text { M6. Los empleos de STEM en su } \\
\text { mayoría están dirigidos a los } \\
\text { hombres. }\end{array}$ & 0.591 & & & & & \\
\hline $\begin{array}{l}\text { M7. Las mujeres deben de trabajar } \\
\text { más en aquellos campos en los que } \\
\text { se trabaje menos horas, ya que sus } \\
\text { actividades como madres les exige } \\
\text { tiempo. }\end{array}$ & 0.583 & & & & & \\
\hline
\end{tabular}




\begin{tabular}{|c|c|c|c|c|c|}
\hline $\begin{array}{l}\text { M8. Las mujeres pocas veces tienen } \\
\text { éxito en la ciencia, ya que es un } \\
\text { campo de los hombres. }\end{array}$ & 0.590 & & & & \\
\hline $\begin{array}{l}\text { M11. Existen trabajos que son } \\
\text { adecuados para las mujeres y para } \\
\text { los hombres. }\end{array}$ & 0.434 & & & & \\
\hline $\begin{array}{l}\text { G15. He tenido logros importantes } \\
\text { en el área de matemáticas durante mi } \\
\text { trayectoria académica. }\end{array}$ & & & 0.352 & & \\
\hline $\begin{array}{l}\text { A16. Cuando obtengo malas } \\
\text { calificaciones en matemáticas, me } \\
\text { presiono y me pongo a estudiar para } \\
\text { resarcir la deficiencia. }\end{array}$ & & 0.351 & & & \\
\hline $\begin{array}{l}\text { A17. Considero que soy autodidacta, } \\
\text { por lo tanto, soy responsable de mis } \\
\text { debilidades académicas. }\end{array}$ & & 0.546 & & & \\
\hline $\begin{array}{l}\text { I19. Alguien cercano trabaja en } \\
\text { algún campo de STEM, de ahí mi } \\
\text { inspiración para estudiar esta carrera. }\end{array}$ & & & & 0.434 & \\
\hline $\begin{array}{l}\text { ES20. La escuela cuenta con clubs y } \\
\text { cursos de temas avanzados de la } \\
\text { carrera que estoy estudiando. }\end{array}$ & & & & & 0.403 \\
\hline $\begin{array}{l}\text { ES21. En la escuela los profesores se } \\
\text { esfuerzan por generar mayor interés } \\
\text { por aquellas asignaturas relacionadas } \\
\text { con los campos de STEM. }\end{array}$ & & & & & 0.747 \\
\hline $\begin{array}{l}\text { I26. Creo que mis padres han tenido } \\
\text { mucha influencia en mi decisión } \\
\text { sobre lo que quiero estudiar. }\end{array}$ & & & & 0.501 & \\
\hline $\begin{array}{l}\text { A10. Durante mis estudios he tenido } \\
\text { contacto con un científico o } \\
\text { científica. }\end{array}$ & & 0.321 & & & \\
\hline
\end{tabular}




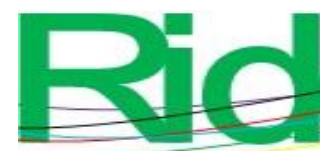

Revista Iberoamericana para la

Investigación y el Desarrollo Educativo

ISSN 2007 - 7467

\begin{tabular}{|l|l|l|l|l|l|l|}
\hline $\begin{array}{l}\text { M12. Los hombres ganan más que } \\
\text { las mujeres en los campos de STEM. }\end{array}$ & 0.685 & & & & & \\
\hline $\begin{array}{l}\text { A13. Los varones pueden llegar a ser } \\
\text { mejores científicos que las mujeres. }\end{array}$ & 0.514 & & & & & \\
\hline $\begin{array}{l}\text { A18. Conozco el campo laboral de lo } \\
\text { que estoy estudiando. }\end{array}$ & & 0.632 & & & & \\
\hline $\begin{array}{l}\text { C25. A veces pienso que los temas } \\
\text { de STEM son para personas } \\
\text { solitarias y con alto nivel intelectual. }\end{array}$ & & & & & 0.334 & \\
\hline $\begin{array}{l}\text { I27. Mis padres benefician a los hijos } \\
\text { varones, dotándolos de beneficios y } \\
\text { apoyando sus decisiones educativas. }\end{array}$ & & & & & 0.447 & \\
\hline
\end{tabular}

Fuente: Elaboración propia

Posteriormente, se trabajó la matriz patrón en el software Atlas Ti versión 23 para obtener el modelo inicial que se muestra en la figura 1. 


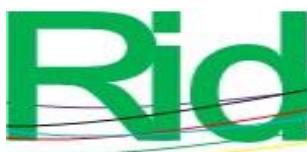

Revista Iberoamericana para la

Investigación y el Desarrollo Educativo ISSN $2007-7467$

Figura 1. Modelo de medida inicial

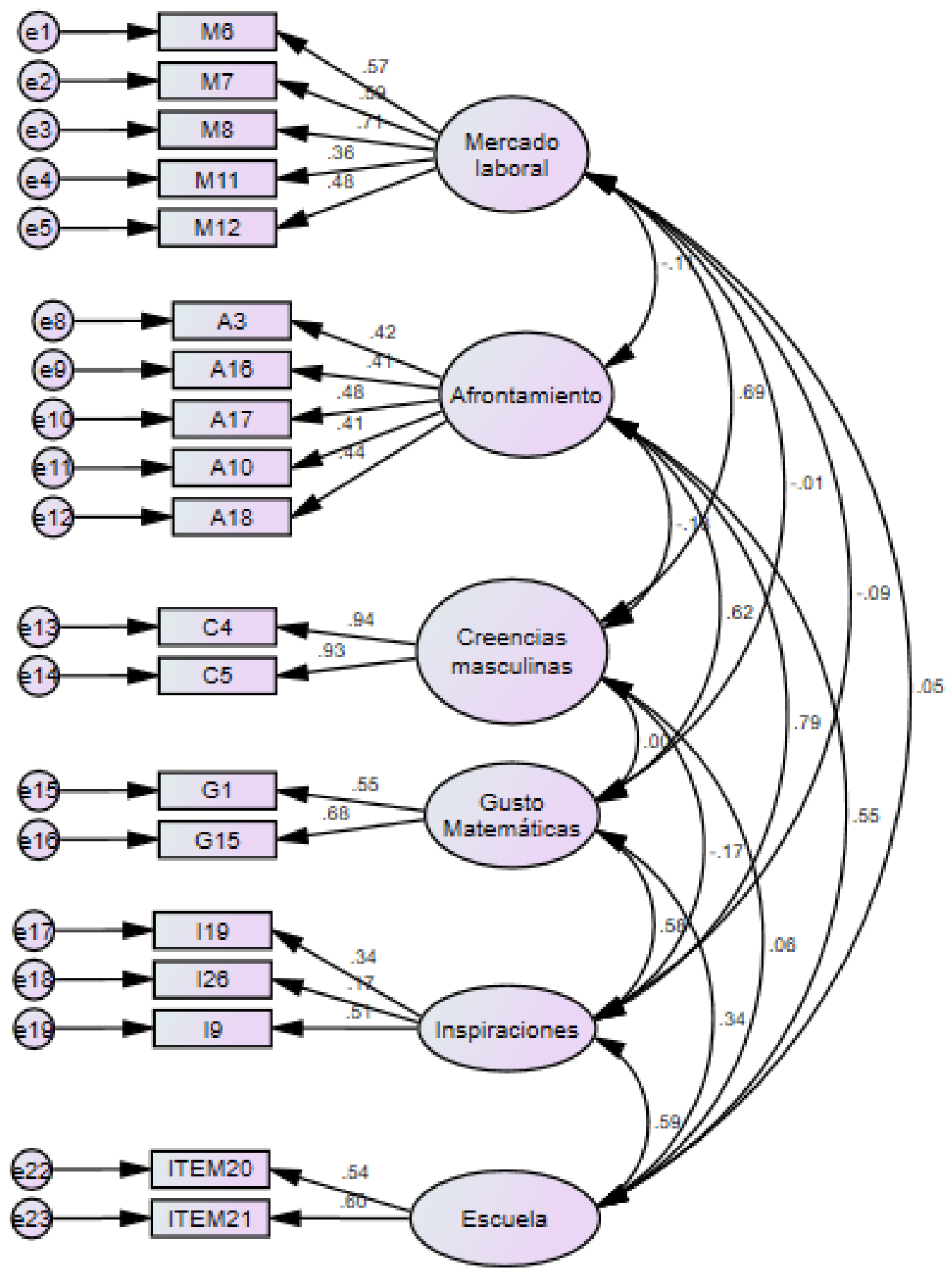

Fuente: Elaboración propia

La tabla 2 muestra las medidas de adecuación del modelo con sus umbrales de adecuación. 


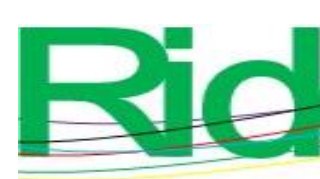

Revista Iberoamericana para la Investigación y el Desarrollo Educativo ISSN $2007-7467$

Tabla 2. Medidas de adecuación del modelo

\begin{tabular}{|l|l|l|l|}
\hline Medida & Estimación & Umbral de aceptación & Interpretación \\
\hline CMIN/DF & 4.535 & Entre 1 y 3 & Aceptable \\
\hline CFI & 0.929 & $>0.95$ & Aceptable \\
\hline SRMR & 0.047 & $<0.08$ & Excelente \\
\hline RMSEA & 0.044 & $<0.06$ & Excelente \\
\hline PClose & 0.997 & $>0.05$ & Excelente \\
\hline
\end{tabular}

Fuente: Elaboración propia

La tabla 2 muestra las medidas de ajuste que son adecuadas para el modelo inicial. Se empleó el modelo de ecuaciones estructurales, un tipo de modelo estadístico multivariante que coadyuva a estimar el efecto y las posibles relaciones entre diversas variables. La utilidad del modelo es que permite establecer las forma y la dirección de las posibles relaciones que se desean encontrar para después estimar los parámetros que vienen especificados en las relaciones que surgen del marco teórico propuesto. Un modelo de este tipo está integrado por dos partes: $a$ ) el modelo de medida y $b$ ) el modelo de relaciones estructurales. En suma, permite evaluar o testear modelos teóricos; es una herramienta poderosa para el análisis de datos no causales (Chahín y Libia, 2018; Ruiz, Pardo y San Martín, 2010). El modelo que ecuaciones estructurales que se propuso para el estudio se presenta en la figura 2. 


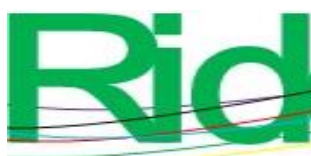

Figura 2. Modelo de ecuaciones estructurales. Estimación por máxima verosimilitud

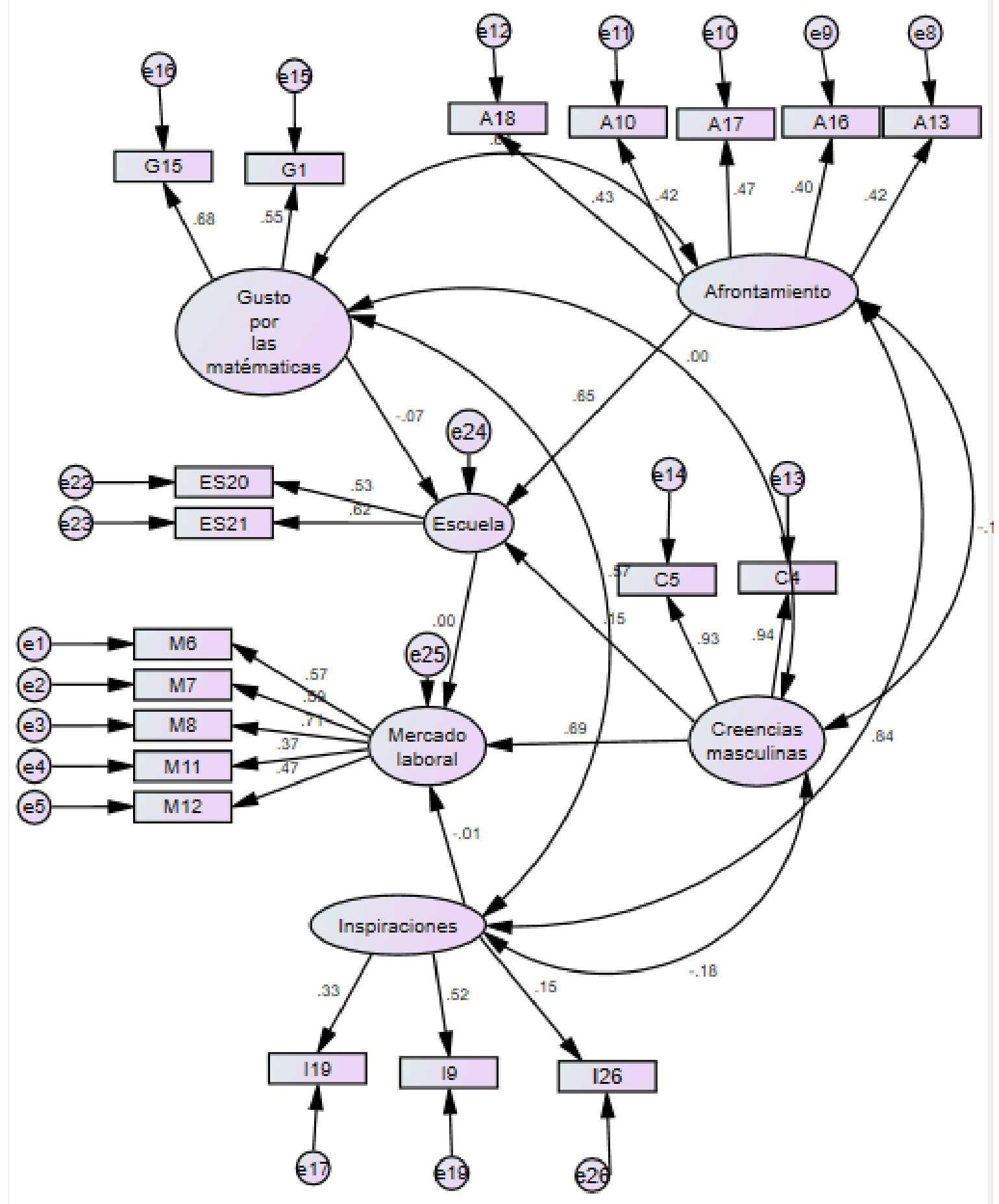

Fuente: Elaboración propia

La tabla 3 expone las hipótesis que se plantearon para este estudio. 


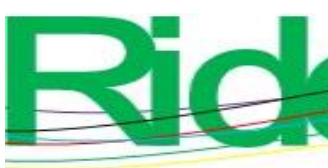

Revista Iberoamericana para la Investigación y el Desarrollo Educativo ISSN $2007-7467$

Tabla 3. Hipótesis planteadas con los estimadores que permiten identificar su aceptación o rechazo

\begin{tabular}{|c|c|c|}
\hline Relaciones causales & Modelo / Estimador & Hipótesis \\
\hline $\begin{array}{l}\text { El gusto por las matemáticas } \\
\text { aporta a la variable Escuela. }\end{array}$ & $\begin{array}{l}-0.042 \\
\text { significativo) }\end{array}$ & $\begin{array}{l}\text { Se rechaza la hipótesis de que el } \\
\text { gusto por las matemáticas aporta } \\
\text { a la variable Escuela. }\end{array}$ \\
\hline $\begin{array}{l}\text { El afrontamiento aporta al } \\
\text { constructo Escuela. }\end{array}$ & $0.384 * *$ & $\begin{array}{l}\text { Se acepta la hipótesis de que el } \\
\text { afrontamiento coadyuva a } \\
\text { explicar la escuela. }\end{array}$ \\
\hline $\begin{array}{l}\text { Las creencias masculinas } \\
\text { aportan a la variable latente } \\
\text { Escuela. }\end{array}$ & $0.086^{* *}$ & $\begin{array}{l}\text { Se acepta la hipótesis de que las } \\
\text { creencias masculinas aportan } \\
\text { positivamente para explicar el } \\
\text { constructo Escuela. }\end{array}$ \\
\hline $\begin{array}{l}\text { Las creencias masculinas } \\
\text { aportan a explicar el mercado } \\
\text { laboral. }\end{array}$ & $0.453 * *$ & $\begin{array}{l}\text { Se acepta la hipótesis de que las } \\
\text { creencias masculinas aportan para } \\
\text { explicar la variable Mercado } \\
\text { Laboral. }\end{array}$ \\
\hline $\begin{array}{l}\text { Las inspiraciones aportan al } \\
\text { mercado laboral. }\end{array}$ & $\begin{array}{l}-0.006 \\
\text { significativo) }\end{array}$ & $\begin{array}{l}\text { Se rechaza la hipótesis de que las } \\
\text { inspiraciones aporten para } \\
\text { explicar el mercado laboral. }\end{array}$ \\
\hline $\begin{array}{l}\text { La escuela aporta al mercado } \\
\text { laboral- }\end{array}$ & $\begin{array}{l}0.004 \\
\text { significativo) }\end{array}$ & $\begin{array}{l}\text { Se rechaza la hipótesis de que la } \\
\text { escuela aporta para estudiar el } \\
\text { mercado laboral. }\end{array}$ \\
\hline
\end{tabular}

Fuente: Elaboración propia

\section{Discusión}

Los resultados encontrados señalan que las variables que integran el afrontamiento coadyuvan a explicar la variable Escuela. Como se sabe, el ambiente de STEM es complejo por la carga de asignaturas relacionadas con las matemáticas, aunado a los contextos hostiles que se desarrollan en las universidades, donde en ocasiones surgen mensajes erróneos y limitan el desarrollo y avance de las mujeres. Por lo anterior, se requiere que los estudiantes desarrollen habilidades para persistir en este campo. De acuerdo con Thiry (2019), los elementos que refuerzan la persistencia de las mujeres en los campos de STEM son: a) los 


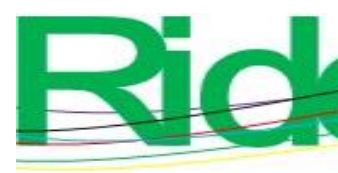

Revista Iberoamericana para la Investigación y el Desarrollo Educativo ISSN $2007-7467$

grupos de estudio, $b$ ) las oportunidades de vinculación con otras mujeres, $c$ ) trabajar con mujeres académicas y mentoras, d) programas de asistencia académica, e) prácticas de asesoramiento y orientación implementadas por las universidades, $f$ ) sostener la voluntad de persistir, $g$ ) que los estudiantes crean en sí mismo, $h$ ) desarrollar hábitos de trabajo eficaces e $i$ ) intentar encontrar sistemas de apoyo. Por otra parte, Holland (2019) añade que las grandes luchas a las que se enfrentan los estudiantes de STEM son: 1) el mapa curricular, 2) resarcir las malas calificaciones y enfrentarse a las diversas prácticas de laboratorios, 3) sentirse que pertenecen a las instituciones y 4) desarrollar persistencia. Watson (2020) añade que las mujeres deben identificar y asimilar los sacrificios de estar en una carrera STEM, al igual que desarrollar habilidades de liderazgo y de comunicación y aprender a tomar decisiones basadas en datos y no en emociones.

En este trabajo también se encontró que las creencias masculinas aportan de forma positiva a explicar la variable Escuela. Las variables que se estudiaron fueron: a) creencia de que los hombres son mejores en las matemáticas que las mujeres y $b$ ) los hombres son mejores científicos que las mujeres. Casad et al. (2019) señalan que las mujeres están conscientes de su estigmatización en los campos STEM, lo que provoca incertidumbre en los entornos académicos: cuando las mujeres perciben amenazas forman juicios negativos sobre de ellas. La incertidumbre desarrolla una cadena de eventos psicológicos que se incrementan con el paso del tiempo. Los resultados encontrados respaldan lo expuesto por Peila (2017), quien menciona que las mujeres tienen que superar una diversidad de obstáculos, muchos de los cuales tienen su origen en los estereotipos de género, que siempre van acompañados de prejuicios individuales e institucionales.

Siguiendo con las creencias masculinas que explican el factor Escuela, de acuerdo con lo expuesto por Allegrini, Pellegrini y Segafredo (2015), los hombres sienten mayor atracción por alcanzar un estatus en el ámbito de STEM. Esto se debe a que los modelos institucionales continúan siendo modelos socioculturales tradicionales en donde la figura masculina es la más representativa. Asimismo, se tiene la percepción de que la ciencia es un entorno masculino. En efecto, los hombres se benefician más de las carreras STEM que las mujeres (Olitsky, 2014). Es lamentable que, a pesar de que las mujeres cuenten con los antecedentes académicos necesarios en los campos STEM, se sigan enfrentando a innumerables obstáculos para llevar a cabo estudios avanzados, sin perder de vista que la carencia de modelos femeninos a seguir, la ausencia del apoyo entre sus pares, posibles interrupciones de carrera por maternidad, las responsabilidades del cuidado, la falta de apoyo, 


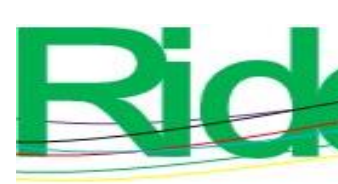

Revista Iberoamericana para la
Investigación y el Desarrollo Educativo
ISSN $2007-7467$

la escasa tutoría de los hombres, el sexismo cotidiano, la violencia sexual y la cultura de misoginia inciden en alejar a las mujeres de estos entornos profesionales (Cuthbert y Sidelil, 2019).

Es imprescindible que las instituciones educativas desarrollen intervenciones para incrementar la representación de mujeres académicas (Casad et al., 2019). Además, deben crear ambientes de igualdad de género para que las mujeres logren avanzar en sus carreras profesionales (Yang y Gao, 2019). Algunas estrategias que pueden coadyuvar al equilibrio de género en los campos de STEM son: a) identificar y promover los modelos de roles femeninos, b) implementar programas para desarrollar mujeres líderes, c) generar oportunidades para que las mujeres incrementen su presencia en los campos científicos, $d$ ) desarrollo de programas de retención y orientación profesional, $e$ ) establecer la tutoría como parte de la formación profesional de las mujeres, $f$ ) trabajar con el cambio de mentalidad de las jóvenes, $g$ ) generar programas de coaching para que las mujeres descubran y mejoren su potencial y $h$ ) desarrollo de habilidades políticas (Barabino et al., 2020).

Las actividades que se pueden desarrollar para alentar a las niñas y mujeres para elegir campos de STEM y de esa forma abatir las creencias masculinas vigentes pueden ser: 1) reconocer y trabajar para desmentir los estereotipos de género que les otorgan a los hombres las habilidades STEM, es decir, "las matemáticas son masculinas”, 2) fomentar en las niñas el interés por las matemáticas y las ciencias, 3) acercamiento temprano de las niñas a las matemáticas, 4) socializar la información a las niñas y mujeres sobre todo el catálogo de carreras STEM y sus campos laborales, 5) campamentos de verano científicos para niñas y mujeres, 6) desarrollar videos que permitan exponer modelos femeninos exitosos en las áreas STEM y 7) alentar a las mujeres y niñas a tomar cursos avanzados en matemáticas y ciencias (Watson, 2020).

Otros elementos que coadyuvan a la persistencia son los grupos de estudio, las oportunidades de vinculación con otras mujeres y trabajar con mujeres académicas y mentoras. La retención se apoya de programas de asistencia académica, asesoramiento y orientación implementados por las universidades (Thiry, 2019). Rosenthal, Levy, London, Lobel y Bazile (2013) agregan que las mujeres universitarias que tienen contacto con mujeres exitosas generan un mayor sentido de pertinencia y muestran un mayor interés por continuar con la carrera. Se sabe que las niñas de sectores con mayores ingresos con el tiempo incrementan sus afinidades científicas, sin embargo, las niñas con familias de bajos recursos disminuyen sus intereses científicos; de ahí que es importante que las escuelas pongan 


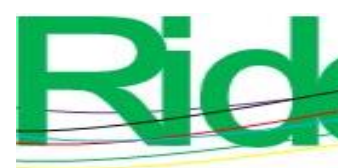

Revista Iberoamericana para la
Investigación y el Desarrollo Educativo ISSN $2007-7467$

estereotipadas que consideran a los varones más aptos para áreas en cuestión. Se tiene que desdibujar las ideas de que los varones son más competentes y dejar de pensar que las mujeres poseen débiles aspiraciones en ese tipo de carreras, dejar de pensar que cuentan con escasa capacidad y que tienen baja autoeficacia. Se sugiere trabajar con talleres de sensibilización con perspectiva de género, así como de implementar estrategias educativas dirigidas a mujeres, conformar una red de apoyo de mentoría que permita que ellas puedan conocer a científicas y profesoras que las puedan orientar y motivar cuando se presenten momentos complicados en la carrera. La escuela tiene que enfocar sus esfuerzos sobre aquellas mujeres que provienen de sectores sociales poco favorecidos y en situación de pobreza, por lo que se debe tener un censo para poder enviar información a las estudiantes sobre becas y programas de apoyo.

Respecto a las creencias masculinas que explican la variable del mercado laboral, se concluye que se necesita de esfuerzos que coadyuven en incentivar a las empresas para que eliminen ideologías de género que impidan el acceso al empleo a mujeres en las áreas STEM. Y trabajar con políticas que impulsen su inclusión y no discriminación por razones de género. Por otra parte, se tienen que eliminar los mitos relacionados con la maternidad. Cuando esta se presenta en las mujeres STEM, es un obstáculo que les impiden continuar en los puestos de trabajo, aunado a las escasas políticas que apoyen a las mujeres a contar con espacios destinados para el cuidado de sus hijos.

\section{Futuras líneas de investigación}

Las futuras líneas de investigación están orientadas a llevar a cabo estudios con mujeres egresadas de los campos STEM para identificar cuáles son los principales obstáculos a los que se han enfrentado, saber si están desarrollándose profesionalmente en áreas STEM, conocer cuántas de ellas siguen con sus trayectorias académicas en los posgrados nacionales e internacionales, saber el tiempo que tienen en el mercado laboral y de esa forma contabilizar las mujeres que han llegado a ocupar puestos estratégicos en los sectores, así como estudiar las barreras que han frenado su desarrollo. 


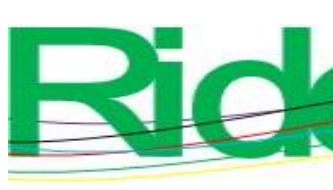

Revista Iberoamericana para la
Investigación y el Desarrollo Educativo ISSN 2007-7467

\section{Referencias}

Acker, J. (2006). Inequality regimes gender, class, and race in organizations. Gender Soc 20(4), 441-464

Allegrini A., Pellegrini G. \&, Segafredo C. (2015) Italian Female and Male Students' Choices: STEM Studies and Motivations. In: Henriksen E., Dillon J., Ryder J. (eds) Understanding Student Participation and Choice in Science and Technology Education. Springer, Dordrecht. https://doi.org/10.1007/978-94-007-7793-4_18

Barabino, G., Frize, M., Ibrahim, F., Kaldoudi, E., Lhotska, L., Marcu, L. and Bezak, E. (2020). Solutions to Gender Balance in STEM Fields Through Support, Training, Education and Mentoring: Report of the International Women in Medical Physics and Biomedical Engineering Task Group. Science and Engineering Ethics, 26(1), 275292.

Barbulescu, R., \& Bidwell, M. (2013). Do women choose different jobs from men? Mechanisms of application segregation in the market for managerial workers. Organization Science, 24(3), 737-756.

Bertocchi G. and Bozzano M. (2020) Gender Gaps in Education. In Zimmermann K. (ed.), Handbook of Labor, Human Resources and Population Economics. Cham, Switzerland: Springer. Retrieved from https://doi.org/10.1007/978-3-319-573656_26-1.

Carli, L. L., Alawa, L., Lee, Y., Zhao, B. and Kim, E. (2016). Stereotypes About Gender and Science: Women $\neq$ Scientists. Psychology of Women Quarterly, 40(2), 244-260. Retrieved from https://doi.org/10.1177/0361684315622645.

Casad, B. J., Petzel, Z. W. and Ingalls, E. A. (2019). A Model of Threatening Academic Environments Predicts Women STEM Majors' Self-Esteem and Engagement in STEM. Sex Roles, 80, 469-488. Retrieved from https://doi.org/10.1007/s11199-0180942-4.

Castaño, A. M., Lubiano, M. A. and García, A. L. (2020). Gendered Beliefs in STEM Undergraduates: A Comparative Analysis of Fuzzy Rating versus Likert Scales. Sustainability, 12(15), 6227.

Chahín, N. y Libia, B. (2018). Propiedades psicométricas del Cuestionario de Adicción a Internet y a los Videojuegos para Adolescentes. Universitas Psychologica, 17(4), 113. 


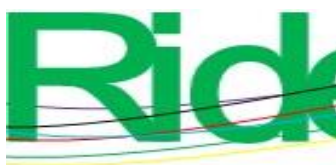

\section{Revista Iberoamericana para la Investigación y el Desarrollo Educativo ISSN $2007-7467$}

Christman, D. E. and McClellan, R. L. (2012). Discovering Middle Space: Distinctions of Sex and Gender in Resilient Leadership. The Journal of Higher Education, 83(5), 648-670. Retrieved from https://www.jstor.org/stable/23256880.

Copur, Y., Thacker, I. and Quinn, D. (2020). K-8 Teachers' Overall and Gender-Specific Beliefs About Mathematical Aptitude. International Journal of Science and Mathematics Education, 1-19. Retrieved from https://doi.org/10.1007/s10763-02010104-7.

Cundiff, J. L., Vescio, T. K., Loken, E. and Lo, L. (2013). Do gender-science stereotypes predict science identification and science career aspirations among undergraduate science majors? Social Psychology of Education, 16(4), 541-554.

Cuthbert, D. and Sidelil, L. T. (2019). Gender Equity Instrumentalism and (Re)Building the Nation Through Innovation: Critical Reflections on Women in STEM Policy in Australia. In Neubauer, D. and Kaur, S. (eds.), Gender and the Changing Face of Higher Education in Asia Pacific. International and Development Education. Cham, Switzerland: Palgrave Macmillan. Retrieved from https://doi.org/10.1007/978-3-03002795-7_5.

De Beauvoir, S. (1981). El segundo sexo. Buenos Aires, Argentina: Siglo XX.

Deb, A. (2018). Psychology of Resilience. In Misra G. (ed.), Psychosocial Interventions for Health and Well-Being (pp. 43-57). New Delhi, India: Springer. Retrieved from https://doi.org/10.1007/978-81-322-3782-2_4.

Eaton, A. A., Saunders, J. F., Jacobson, R. K. and West, K. (2020). How Gender and Race Stereotypes Impact the Advancement of Scholars in STEM: Professors' Biased Evaluations of Physics and Biology Post-Doctoral Candidates. Sex Roles, 82, 127141. Retrieved from https://doi.org/10.1007/s11199-019-01052-w

Foro Económico Mundial [WEF, por sus siglas en inglés]. (2020). Global Gender Gap Report 2020. Geneva, Switzerland: Foro Económico Mundial.

Galperin, H. (2019). “This Gig Is Not for Women”: Gender Stereotyping in Online Hiring. Social Science Review. https://doi.org/10.1177/0894439319895757.

Grau, K. and Martinez, A. (2017). Women's interest development and motivations to persist as college students in STEM: a mixed methods analysis of views and voices from a Hispanic-Serving Institution. International Journal of STEM Education, 4. Retrieved from https://doi.org/10.1186/s40594-017-0059-2. 


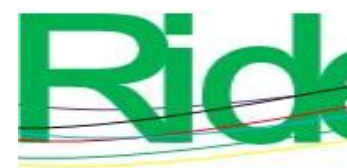

\section{Revista Iberoamericana para la Investigación y el Desarrollo Educativo ISSN $2007-7467$}

Achievement Levels. Research in Higher Education, 60, 577-605. Retrieved from https://doi.org/10.1007/s11162-018-9523-1.

Miluka, J. (2013). Not Just Education: The Gender Wage Gap in the Albanian Labor Markets Through Occupational Segregation, Work Experience, and Child Care. In Ruggeri, C. and Savastano, S. (eds.), Poverty and Exclusion in the Western Balkans (pp. 155176). New York, United States: Springer. Retrieved from https://doi.org/10.1007/978-1-4614-4945-4_10.

Moss, C. A., Sanzari, C., Caluori, N. and Rabasco, H. (2018). Gender bias produces gender gaps in STEM engagement. Sex Roles, 79(11-12), 651-670.

Murphy, S., MacDonald, A., Wang, C. A. and Danaia, L. (2019). Towards an Understanding of STEM Engagement: A Review of the Literature on Motivation and Academic Emotions. Canadian Journal of Science, Mathematics and Technology Education, 19, 304-320. https://doi.org/10.1007/s42330-019-00054-w

Okahana, H. and Hao, Y. (2019). Are They Worth it?: Master's Degrees and Labor Market Outcomes in the STEM Workforce. Innovative Higher Education, 44, 165-185. Retrieved from https://doi.org/10.1007/s10755-019-9455-5.

Olitsky, N. H. (2014). How Do Academic Achievement and Gender Affect the Earnings of STEM Majors? A Propensity Score Matching Approach. Research in Higher Education, 55, 245-271. Retrieved from https://doi.org/10.1007/s11162-013-9310-y.

Organización de las Naciones Unidas para la Educación, la Ciencia y la Cultura [Unesco]. (2019). Descifrar el código: la educación de las niñas y las mujeres en ciencias, tecnología, ingeniería y matemáticas (STEM). Francia: Organización de las Naciones Unidas para la Educación, la Ciencia y la Cultura. Recuperado de https://unesdoc.unesco.org/ark:/48223/pf0000366649.

Paunonen, S. V. and Hong, R. Y. (2010). Self-efficacy and the Prediction of Domain-Specific Cognitive Abilities. Journal of Personality, 78(1), 339-360. Retrieved from https://doi.org/10.1111/j.1467-6494.2009.00618.x.

Peila, J. J. (2017). Women's Career Construction: Promoting Employability Through Career Adaptability and Resilience. In Maree, K. (ed.), Psychology of Career Adaptability, Employability and Resilience. Cham: Springer. Retrieved from https://doi.org/10.1007/978-3-319-66954-0_17. 

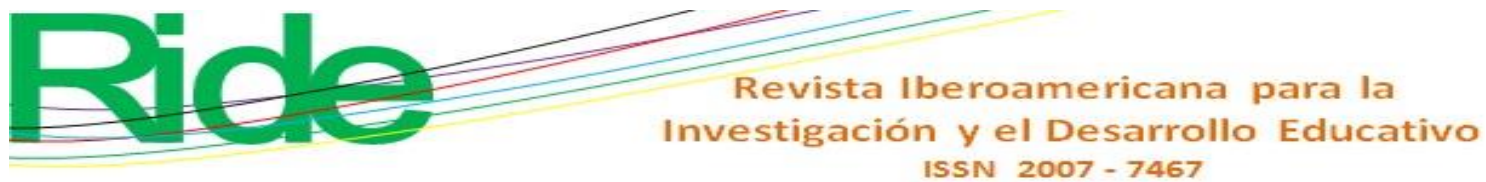

Pološki, N., Obadić, A. and Sinčić, D. (2019). Gender Inequality: Specific Areas of Gender Segregation. In Gender Equality in the Workplace (pp. 37-60). Cham, Switzerland: Palgrave Pivot. Retrieved from https://doi.org/10.1007/978-3-030-18861-0_3.

Poniszewska A., Szukalska A. and Wilczyński, Ł. (2021). Learner-Centred Pedagogical Approach Based on Smart City Concept to Enhance the Development of STEM Skills of Children. In Barolli, L., Poniszewska, A. and Enokido, T. (eds.), Complex, Intelligent and Software Intensive Systems. CISIS 2020. Advances in Intelligent Systems and Computing. Cham, Switzerland: Springer. Retrieved from https://doi.org/10.1007/978-3-030-50454-0_20.

Del Pozo, E., de la Puente, M. A., Fernández, J. A., Belope, S., Rodríguez, E. and Escot, L. (2020). Whether your name is Manuel or María matters: gender biases in recommendations to study engineering. Journal of Gender Studies, 29(7), 805-819.

Rebollo, Y. F. and De la Rica, S. (2020). Gender gaps in skills and labor market outcomes: evidence from the PIAAC. Review of Economics of the Household. Retrieved from https://doi.org/10.1007/s11150-020-09523-w.

Rosenthal, L., Levy, S. R., London, B., Lobel, M. and Bazile, C. (2013). In pursuit of the MD: The impact of role models, identity compatibility, and belonging among undergraduate women. Sex Roles, 68, 464-473. Retrieved from https://doi.org/10.1007/s11199-012-0257-9.

Ruiz, M. A., Pardo, A. y San Martín, R. (2010). Modelos de ecuaciones estructurales. Papeles del Psicólogo, 31(1), 34-45.

Sax, L. J., Kanny, M. A., Riggers, T. A., Whang, H. and Paulson, L. N. (2015). "But I'm Not Good at Math": The Changing Salience of Mathematical Self-Concept in Shaping Women's and Men's STEM Aspirations. Research in Higher Education, 56, 813842. Retrieved from https://doi.org/10.1007/s11162-015-9375-x.

Schlenker, E. (2015). The labour supply of women in STEM. IZA Journal of European Labor Studies, 4. Retrieved from https://doi.org/10.1186/s40174-015-0034-1.

Skolnik, J. (2015). Why Are Girls and Women Underrepresented in STEM, and What Can Be Done About It? Science \& Education, 24, 1301-1306. Retrieved from https://doi.org/10.1007/s11191-015-9774-6.

Szelényi, K. and Inkelas, K. K. (2011). The Role of Living-Learning Programs in Women's Plans to Attend Graduate School in STEM Fields. Research in Higher Education, 52, 349-369. Retrieved from https://doi.org/10.1007/s11162-010-9197-9. 


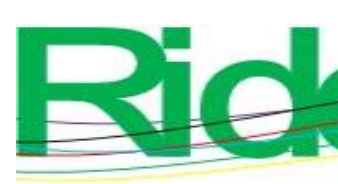

Revista Iberoamericana para la
Investigación y el Desarrollo Educativo
ISSN $2007-7467$

Tellhed, U., Bäckström, M. and Björklund, F. (2017). Will I Fit in and Do Well? The Importance of Social Belongingness and Self-Efficacy for Explaining Gender Differences in Interest in STEM and HEED Majors. Sex Roles, 77, 86-96. Retrieved from https://doi.org/10.1007/s11199-016-0694-y.

Thiry, H. (2019) What Enables Persistence? In Seymour, E. and Hunter, A. B. (eds), Talking about Leaving Revisited (pp. 399-436). Cham, Switzerland: Springer. Retrieved from https://doi.org/10.1007/978-3-030-25304-2_12.

Todd, B. and Zvoch, K. (2019). Exploring Girls' Science Affinities Through an Informal Science Education Program. Research in Science Education, 49, 1647-1676. Retrieved from https://doi.org/10.1007/s11165-017-9670-y.

Valera, N. (2019). Feminismo para principiantes. Penguin Random House.

van Langen, A. (2015). Girls and STEM Choice in Dutch Education: The Strong Gender Segregation and the Good Practice of the Stimulation Policy. Gender Studies and Research, 13, 26-39.

van Tuijl, C. and van der Molen, J. H. W. (2016). Study choice and career development in STEM fields: an overview and integration of the research. International Journal of Technology and Design Education, 26, 159-183. Retrieved from https://doi.org/10.1007/s10798-015-9308-1.

van Veelen, R., Derks, B. and Endedijk, M. D. (2019). Double Trouble: How Being Outnumbered and Negatively Stereotyped Threatens Career Outcomes of Women in STEM. Frontiers in Psychology, 10, 150. Retrieved from https://doi.org/10.3389/fpsyg.2019.00150.

Wang, M. T. and Degol, J. L. (2017). Gender Gap in Science, Technology, Engineering, and Mathematics (STEM): Current Knowledge, Implications for Practice, Policy, and Future Directions. Educational Psychology Review, 29(1), 119-140.

Watson, S. W. (2020) Resiliency and Women: The Journey to Academic STEM Leadership. In Craig, C. J., Turchi, L. and McDonald, D. M. (eds.), Cross-Disciplinary, CrossInstitutional Collaboration in Teacher Education. Palgrave Studies on Leadership and Learning in Teacher Education. Cham, Switzerland: Palgrave Macmillan. Retrieved from https://doi.org/10.1007/978-3-030-56674-6_11.

Weeden, K. A., Gelbgiser, D., \& Morgan, S. L. (2020). Pipeline Dreams: Occupational Plans and Gender Differences in STEM Major Persistence and Completion. Sociology of Education. https://doi.org/10.1177/0038040720928484. 

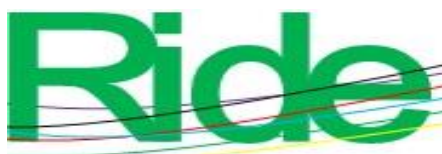

Revista Iberoamericana para la Investigación y el Desarrollo Educativo ISSN 2007 - 7467

Yamaguchi, K. (2019). Impediments to the Advancement of Women in the Japanese Employment System: Theoretical Overview and the Purpose of This Book. In Gender Inequalities in the Japanese Workplace and Employment. Advances in Japanese Business and Economics. Singapore: Springer.

Yang, X. and Gao, C. (2019). Missing Women in STEM in China: an Empirical Study from the Viewpoint of Achievement Motivation and Gender Socialization. Research in Science Education. Retrieved from https://doi.org/10.1007/s11165-019-9833-0.

Zander, L., Höhne, E., Harms, S., Pfost, M., \& Hornsey, M. J. (2020). When Grades Are High but Self-Efficacy Is Low: Unpacking the Confidence Gap Between Girls and Boys in Mathematics. Frontiers in psychology, 11, 2492. 